\title{
Constructing new periodic exact solutions of evolution equations
}

\author{
Jian-min Mao, ${ }^{1}$ Liu Zengrong, ${ }^{1,2}$ and Cao Yongluo ${ }^{1,3}$ \\ ${ }^{1}$ Department of Mathematics, Hong Kong University of Science and Technology, Clearwater Bay, Kowloon, Hong Kong \\ ${ }^{2}$ Department of Mathematics, Shanghai University, Shanghai 201800, People's Republic of China \\ ${ }^{3}$ Department of Mathematics, Suzhou University, Jiangsu, Suzhou 215006, People's Republic of China
}

(Received 8 March 1999)

\begin{abstract}
For the nonlinear Schrödinger equation, the Korteweg-de Vries equation, and the modified Korteweg-de Vries equation, periodic exact solutions are constructed from their stationary periodic solutions, by means of the Bäcklund transformation. These periodic solutions were not written down explicitly before to our knowledge. Their asymptotic behavior when $t \rightarrow-\infty$ is different from that when $t \rightarrow \infty$. Near $t=0$, the spatialtemporal pattern can change abruptly, and rational solitons can appear randomly in space and time. They correspond to new types of "homoclinic orbits" due to different asymptotic behaviors in time.
\end{abstract}

[S1063-651X(99)01310-0]

PACS number(s): 02.30.Jr, 05.45.- a, 42.65.Tg, 42.81.Dp

\section{INTRODUCTION}

As a hot spot in the research field of nonlinear dynamics and chaos, complicated spatial-temporal behavior described by nonlinear evolution equations has been studied intensively in recent years. The evolution equations have been investigated in many different directions, such as numerical simulation, qualitative analysis, perturbation analysis, and search for exact solutions [1]. In the search for exact solutions for a wide range of integrable nonlinear evolution equations, many methods have been developed: the inverse scattering method [2], the Bäcklund transformation [3-5], the Painleve analysis [6,7], the Lie group method [8], the direct algebraic method [9], the tangent hyperbolic method [10], etc. Most of these methods have been used to find exact solutions for evolution equations with a boundary condition at infinity.

The need to find periodic exact solutions of evolution equations has significantly increased recently. For instance, the series of beautiful works by Wiggins and coauthors on homoclinic orbits, multijumped impulse orbits, and chaos in high-dimensional or infinite-dimensional dynamical systems are on systems under a periodic boundary condition $[11,12]$. Some periodic exact solutions of some evolution equations have already been found. For example, a family of periodic solutions of the nonlinear Schrödinger (NLS) equation have been found by assuming the solution is a special combination of a time-independent function, a space-independent function, and a function of both time and space [13].

In this paper, we construct new periodic exact solutions of an integrable nonlinear evolution equation from its stationary periodic solutions by using the Bäcklund transformation and the associated Ablowitz-Kaup-Newell-Segur (AKNS) system. Using this method, we find new periodic exact solutions analytically for the NLS equation, the Korteweg-de Vries $(\mathrm{KdV})$ equation, and the modified Korteweg-de Vries $(\mathrm{MKdV})$ equation. These periodic solutions have not been explicitly written down previously, to our knowledge, and therefore are called new solutions. The new solutions have interesting features. They are kink solutions (i.e., solutions whose asymptotic behavior when $t \rightarrow \infty$ is different from that when $t \rightarrow-\infty)$. Their spatial-temporal pattern changes, gradually or abruptly, near $t=0$. The new periodic solutions correspond to new types of homoclinic orbits with different asymptotic behaviors when time tends to plus or minus infinity.

The method of constructing periodic solutions is described in Sec. II, and applied to the NLS equation in Sec. III, the KdV equation in Sec. IV, and the MKdV equation in Sec V.

\section{THE METHOD}

Let $q(x, t)$ be a known solution of an integrable evolution equation, then a new solution, denoted by $\widetilde{q}(x, t)$, can be obtained by using the following Bäcklund transformation [3]:

$$
\tilde{q}(x, t)=q(x, t)+W(\eta, \Gamma(x, t)),
$$

where $W$ is a function whose form depends on the form of the evolution equation, $\eta$ is a constant, and $\Gamma$ is given by the solution of the following AKNS system associated to the original evolution equation:

$$
\partial_{x} \Phi=\left(\begin{array}{cc}
\eta & q \\
-r & \eta
\end{array}\right) \Phi, \quad \partial_{t} \Phi=\left(\begin{array}{cc}
A & B \\
C & -A
\end{array}\right) \Phi .
$$

Here $\Phi(x, t)=\left(\phi_{1}(x, t), \phi_{2}(x, t)\right)^{T}$ is the unknown function ( $T$ denotes transpose), $r$ (and $q$ ) is a function of $x$ and $t$, and $A, B$, and $C$ are functions of $q, r$, and $\eta$. These functions are chosen so that the original evolution equation can be derived from $\Phi_{x t}=\Phi_{t x}$. The function $\Gamma(x, t)$ in Eq. (1) is given by $\Gamma=\phi_{1} / \phi_{2}$.

In this paper, we focus on how to construct a periodic solution from a known stationary periodic solution. In other words, $q(x, t)=q(x)$, and $q(x)$ and $\tilde{q}(x, t)$ are periodic in $x$. Denote the period by $L$. We consider the case where the functions in the AKNS system, $r, A, B$, and $C$, are all time independent and periodic in $x$ with period $L$. So, Eq. (2) and 
the identity $\Phi_{x t}=\Phi_{t x}$ imply that $\phi_{2}(x, t)$ satisfies $C \phi_{2 x}$ $-r \phi_{2 t}=\frac{1}{2} C_{x} \phi_{2}$, which can be solved by the method of characteristics. Therefore the solution of the AKNS system can be written as

$$
\begin{gathered}
\phi_{1}(x, t)=-\frac{1}{\sqrt{C}}\left[F^{\prime}(\xi)-A F(\xi)\right], \\
\phi_{2}(x, t)=\sqrt{C} F(\xi),
\end{gathered}
$$

where $F$ is a function of $\xi$ and

$$
\xi=\sigma(x)-t, \quad \sigma(x)=-\int_{0}^{x} \frac{r(y)}{C(y)} d y .
$$

Substituting Eq. (3) into Eq. (2), we find that $F(\xi)$ satisfies

$$
F^{\prime \prime}(\xi)-\alpha(\eta) F(\xi)=0,
$$

where

$$
\alpha(\eta)=A^{2}+B C
$$

depends on neither $x$ nor $t$. Here we have used $(d / d x)\left(A^{2}\right.$ $+B C)=0$ because of $\Phi_{x t}=\Phi_{t x}$, and of periodicity and time independence of the functions $A, B$, and $C$. Hence,

$$
\Gamma(x, t)=-\frac{1}{C}[f(\xi)-A],
$$

where $f(\xi)=F^{\prime}(\xi) / F(\xi)$ is given by

$$
f(\xi)=\left\{\begin{array}{cl}
\frac{1}{\xi+c_{0}}, & \text { if } \alpha=0 \\
-\omega \tan \left(\omega \xi+c_{0}\right) & \text { if } \alpha \neq 0, \omega=\sqrt{-\alpha(\eta)} .
\end{array}\right.
$$

Here $c_{0}$ is a constant of integration. If the evolution equation is to be solved in the real space, then, for the case of $\alpha$ $\neq 0, f(\xi)$ is simplified to the tangent function of $\xi$ if $\alpha<0$, and to the hyperbolic tangent function of $\xi$ if $\alpha>0$. On the other hand, if the evolution equation is to be solved in the complex space, then $\omega, \xi$, and $c_{0}$ are all complex and can be written as $c_{0}=c_{1}+i c_{2}, \omega=\omega_{1}+i \omega_{2}$ and $\xi=\xi_{1}+i \xi_{2}$. Therefore, for the case of $\alpha \neq 0, f(\xi)=-\omega\left(\sin u_{1} \cosh u_{2}\right.$ $\left.+i \cos u_{1} \sinh u_{2}\right) /\left(\cos u_{1} \cosh u_{2}-i \sin u_{1} \sinh u_{2}\right)$, where $u_{1}=\omega_{1} \xi_{1}-\omega_{2} \xi_{2}+c_{1}$ and $u_{2}=\omega_{1} \xi_{2}+\omega_{2} \xi_{1}+c_{2}$ are real. And the derivative of $f(\xi)$ is $f^{\prime}(\xi)=-\omega /\left(\cos u_{1} \cosh u_{2}\right.$ $\left.-i \sin u_{1} \sinh u_{2}\right)^{2}$. We also note that, because of the tangent function in Eq. (8), the new solution (1) may be singular.

Examining the expression (1) for the new solution, it is easy to see that if $f(\xi)$ is periodic in $x$ then the new solution is periodic because all other functions in Eq. (1) are periodic, and $f(\xi)$ is periodic if one of the following is true. (i) $\xi$ is periodic in $x$. That is, $\sigma(x)$ given in Eq. (4) is periodic, or, because of the periodicity of $r(x)$ and $C(x)$,

$$
\beta(\eta)=0,
$$

where

$$
\beta(\eta)=-\sigma(L)=\int_{0}^{L} \frac{r(x)}{C(x)} d x .
$$

Here we note that the function $C(x)$ takes $\eta$ as a parameter, as defined in the AKNS system. (ii) $\xi$ is not periodic, but the evolution equation is to be solved in the real space and

$$
\beta(\eta) \sqrt{-\alpha(\eta)}=\frac{n \pi}{L} \text { and } \alpha(\eta)<0
$$

where $n$ is a nonzero integer. In this case, $f(\xi)$ is the tangent function of $\xi$, which is periodic, of course.

It is clear now that in order to have the new solution (1) periodic, $\Gamma(x, t)$ should be as given in Eq. (7), and $\eta$ should satisfy either condition (9) or (11). Using such $\eta$ and $\Gamma$, a new periodic solution can be constructed in the form of Eq. (1). In the following sections, we apply this method of constructing new periodic solutions to the "universal"' nonlinear evolution equations: the NLS, the $\mathrm{KdV}$, and the $\mathrm{MKdV}$ equation.

\section{THE NLS EQUATION}

The NLS equation can be written as

$$
i q_{t}+q_{x x}+2|q|^{2} q=0, \quad 0 \leqslant x \leqslant L, \quad-\infty<t<\infty
$$

where $q(x, t)$ is a complex function. As shown in Appendix $\mathrm{A}$, the equation has the following stationary solution with two parameters, $\gamma$ and $h$, when $h>3\left(\gamma^{4} / 2\right)^{2 / 3}$ :

$$
\begin{aligned}
q(x)= & {\left[a \operatorname{cn}^{2}(\sqrt{a-c} x, k)+b \operatorname{sn}^{2}(\sqrt{a-c} x, k)\right]^{1 / 2} } \\
& \times \exp \left[i\left(\frac{\gamma^{2}}{a \sqrt{a-c}} \Pi(\psi, m, k)+c_{1}\right)\right],
\end{aligned}
$$

where, in the amplitude part, $\operatorname{cn}(\cdot, \cdot)$ and $\operatorname{sn}(\cdot, \cdot)$ are Jacobian elliptic functions, $a=2 \sqrt{h / 3} \cos (\theta / 3), \quad b$ $=2 \sqrt{h / 3} \cos [(4 \pi+\theta) / 3]$, and $c=2 \sqrt{h / 3} \cos [(2 \pi+\theta) / 3]$ for $\theta$ given by $-\gamma^{4}=2(h / 3)^{3 / 2} \cos \theta$ and $\pi / 2<\theta<\pi$, and $k$ $=\sqrt{(a-b) /(a-c)}$ for $0<k<\frac{1}{2}$. In the phase, $\Pi(\cdot, \cdot, \cdot)$ is the normal elliptic integral of the third kind, $\psi$ $=\operatorname{am}(\sqrt{a-c} x, k)$ [function $\operatorname{am}(\cdot, \cdot)$ is the amplitude function], $m=(a-b) / a$, and $c_{1}$ is a constant of integration. This solution is periodic with period $L$ if

$$
2 K(k)=L \sqrt{a-c},
$$

where $K(\cdot)$ is the complete elliptic integral of the first kind. The stationary periodic solution for $\gamma=0.05$ is shown in Fig. 1(a). [The other parameter $h$ is determined by Eq. (14).] The system length is $L=10$ in this figure, and in all other figures in this paper.

The functions in the AKNS system for the NLS equation are [3]

$$
\begin{gathered}
r=-q^{*}, \quad A=2 i \eta^{2}+i|q|^{2}, \\
B=i q_{x}+2 i q, \quad C=i q_{x}^{*}-2 i \eta q^{*},
\end{gathered}
$$

where $*$ denotes complex conjugate. Therefore $\sigma(x)$ is given by 

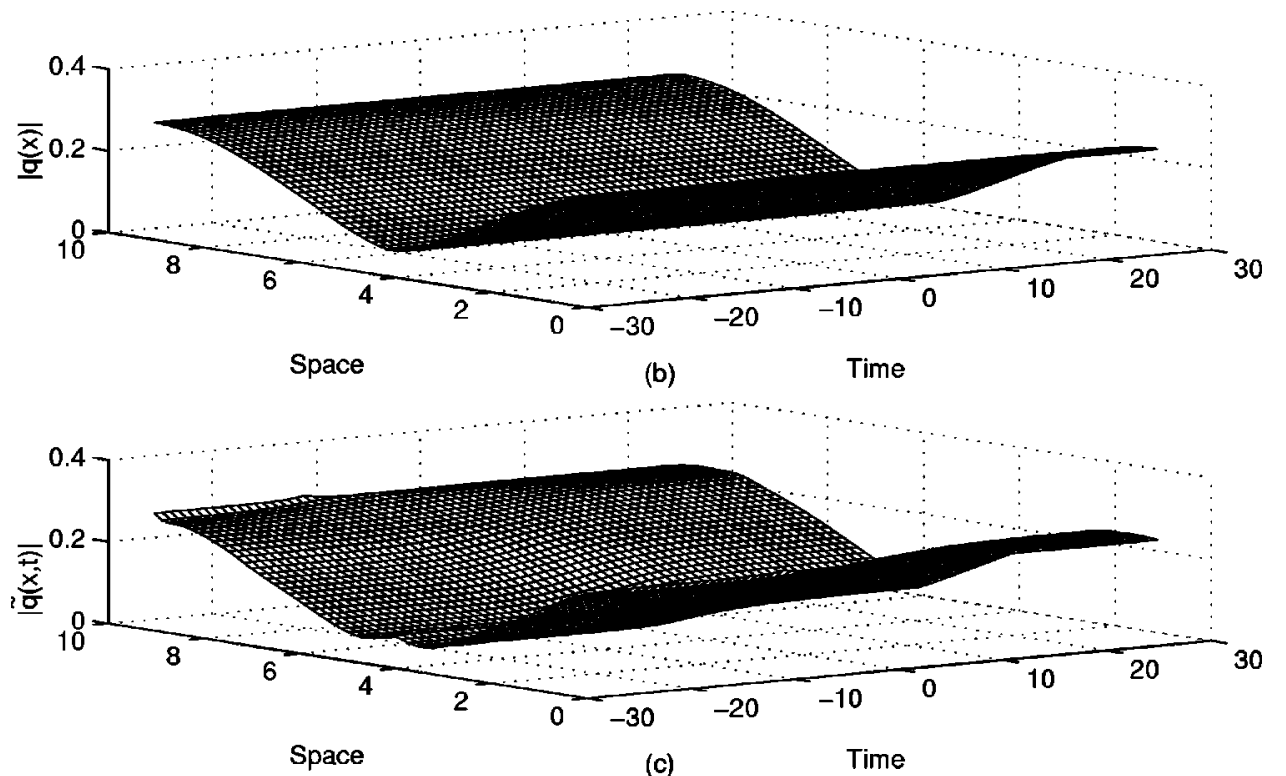

FIG. 1. Periodic solutions of the NLS equation for the constant of integration $\gamma=0.05$. (a) The stationary periodic solution. (b) The periodic solution constructed from the stationary periodic solution shown in (a) when $\eta=-0.0195686 i . \quad \alpha$ is real and $\alpha<0$. (c) $\eta= \pm 1.01877$ $-0.271375 i$. (d) $\eta= \pm 0.459084$ $-0.0513864 i$. In both (c) and (d), $\alpha(\eta)$ are complex.
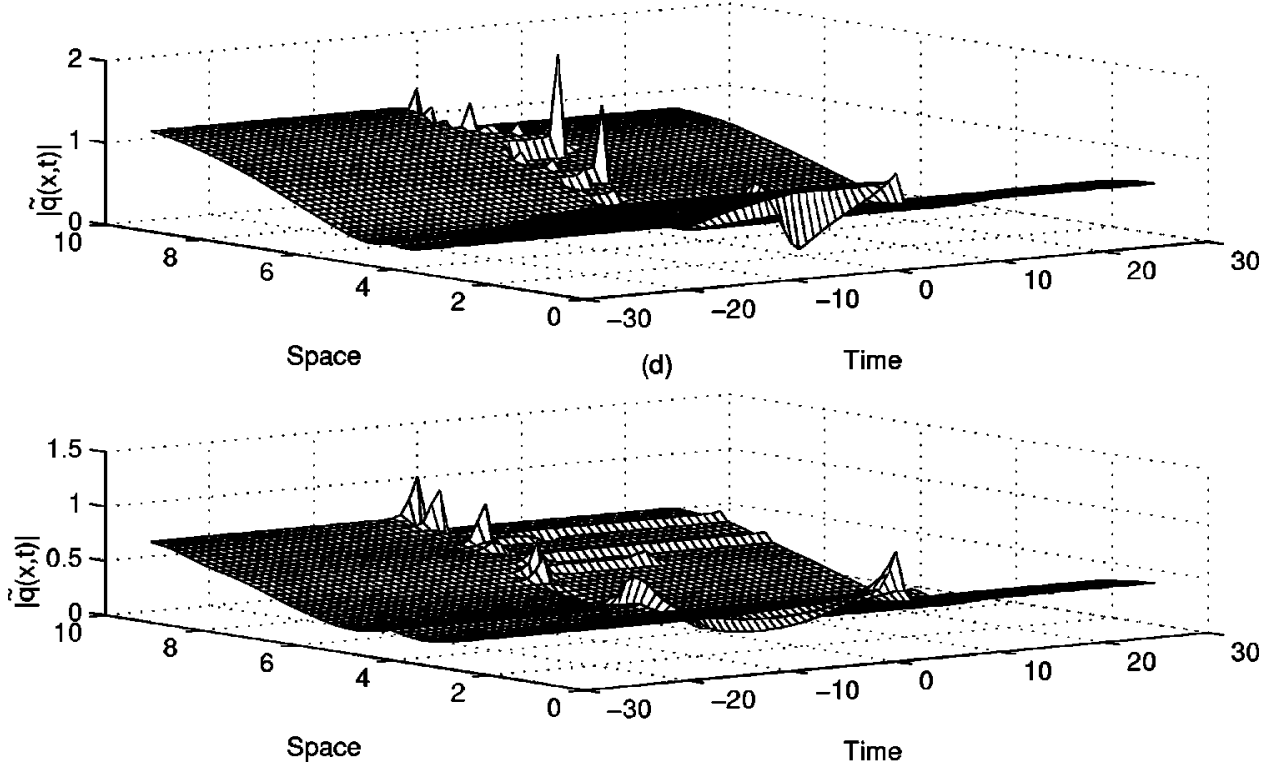

$$
\sigma(x)=i \int \frac{a \operatorname{cn}^{2}(X, k)+b \operatorname{sn}^{2}(X, k)}{(a-b) \operatorname{cn}(X, k) \operatorname{sn}(X, k) \operatorname{dn}(X, k)+2 \eta(a-b) \operatorname{cn}^{2}(X, k)+\left(i \gamma^{2}+2 \eta b\right)} d x,
$$

where $X=\sqrt{a-c} x$. Further, we have

$$
\alpha(\eta)=-4 \eta^{2}-i 4 \eta \gamma^{2}-2 h
$$

The Bäcklund transformation for the NLS equation is [3]

$$
\tilde{q}(x, t)=q(x)-\frac{4 \eta \Gamma}{1+|\Gamma|^{2}}
$$

Substituting the stationary periodic solution (13) for the function $q(x)$ in this transformation, Eq. (7) for the function $\Gamma$, and taking a value of $\eta$ satisfying either Eq. (9) or Eq. (11), this transformation gives a new periodic solution of the NLS equation.
Figures 1(b), 1(c), and 1(d) show the periodic solutions constructed from the stationary periodic solution shown in Fig. 1(a), with different values of $\eta$ satisfying $\beta(\eta)=0$. All these solutions are kink solutions (i.e., having different asymptotes). In Fig. 1(b), $\eta$ is purely imaginary. So, $\alpha$ is real and $\alpha<0$ and the solution is given by a trigonometric function. The solution is oscillatory. The spatial-temporal pattern when $t \rightarrow-\infty$ and that when $t \rightarrow \infty$ are different from each other. Therefore the pattern has to change at some time. The pattern changes gradually near $t=0$. Figure $1(\mathrm{c})$ is for $\eta$ being a complex number with both the real part and the imaginary part nonzero. Therefore $\alpha$ is also complex and the solutions are exponentials. We see from the figure that the $x$ dependence of the solution changes suddenly near $t=0$, and a number of rational solitons appear "randomly" in space 
(a)

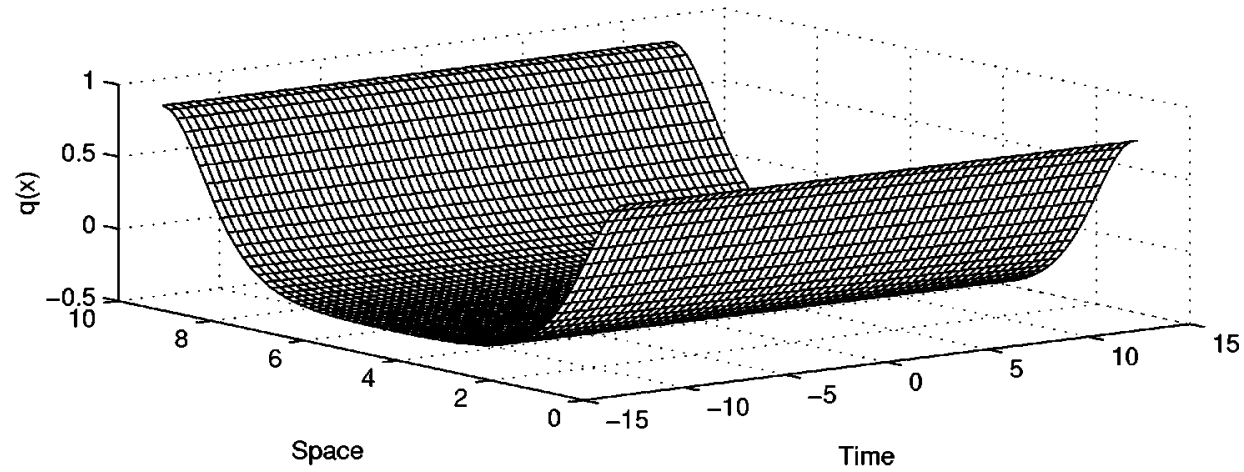

(b)

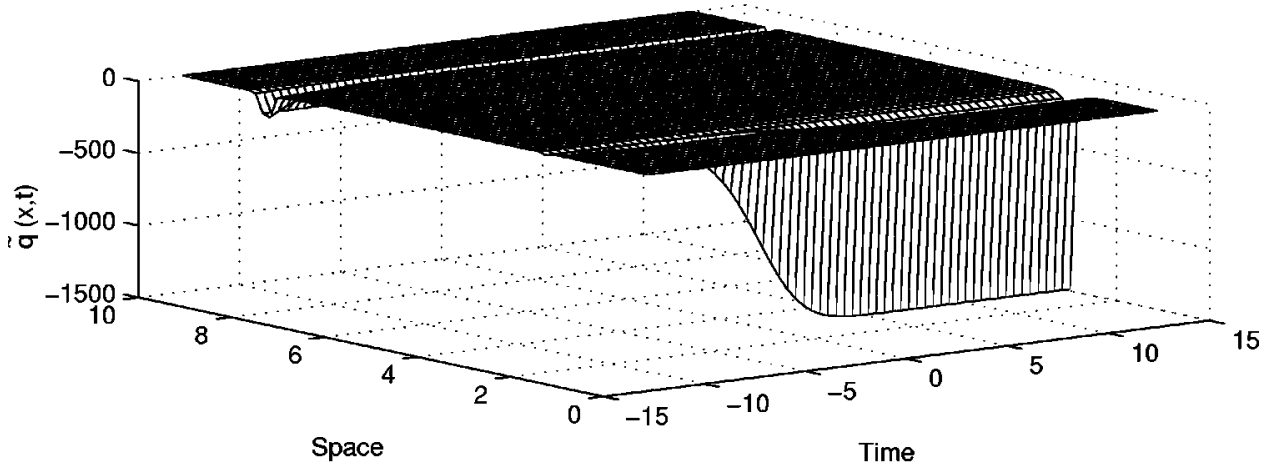

(c)

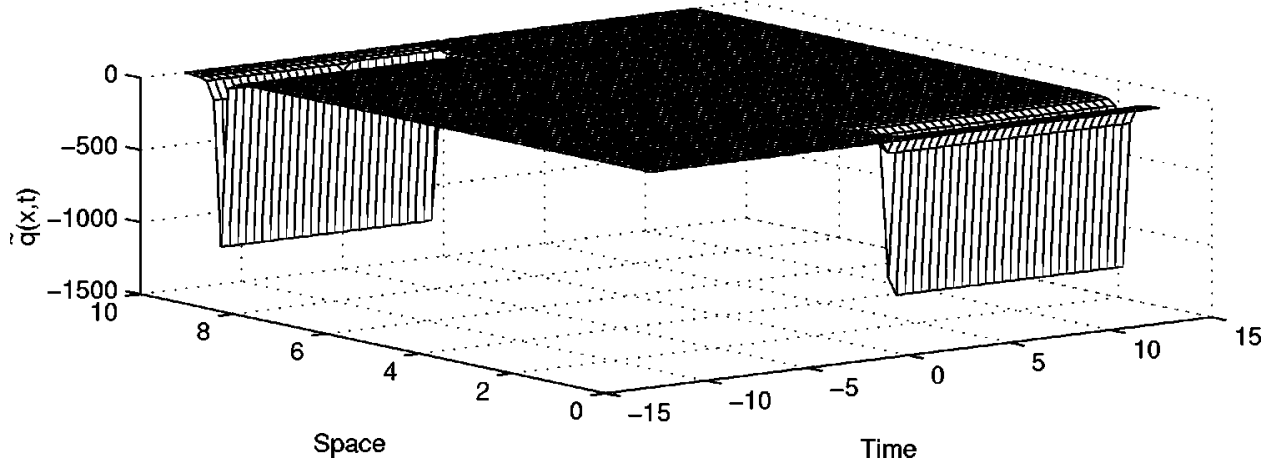

FIG. 2. Periodic solutions of the KdV equation. (a) The stationary periodic solution for the constant of integration $\gamma=0.5$. (b) The periodic solution constructed from the stationary periodic solution shown in (a) when $\eta$ $= \pm 0.2833 . \alpha(\eta)$ is real and $\alpha$ $>0$. (c) The periodic solution constructed from the stationary periodic solution for $\gamma=10$. $\eta$ $= \pm 0.3325$ and $\alpha>0$. and time. Figure 1(d) is for another complex value of $\eta$. The spatial-temporal pattern is similar to Figure 1(c) except that the solution when $t \rightarrow \infty$ is solitary [oscillatory in Fig. 1(c)]. [In our numerical calculation, we could not find a value of $\eta$ satisfying both $\beta(\eta)=0$ and $\alpha(\eta)=0$. Further analytical and numerical works are needed.]

The solutions shown in Figs. 1(b), 1(c), and 1(d) were not explicitly written down before, to our knowledge, and therefore are called new solutions. Each of them has an asymptotic behavior when $t \rightarrow+\infty$ different from that when $t \rightarrow-\infty$. Therefore they correspond to new types of homoclinic orbits due to different asymptotic behaviors in time.

\section{THE KdV EQUATION}

The KdV equation is

$$
q_{t}+6 q q_{x}+q_{x x x}=0, \quad 0 \leqslant x \leqslant L, \quad-\infty<t<\infty
$$

where $q(x, t)$ is a real function. As shown in Appendix B, the equation has the following stationary solution with two parameters, $\gamma>0$ and $h<2(\gamma / 3)^{3 / 2}$ :

$$
q(x)=a \mathrm{cn}^{2}\left(\sqrt{\frac{a-c}{2}} x, k\right)+b \operatorname{sn}^{2}\left(\sqrt{\frac{a-c}{2}} x, k\right),
$$

where $\quad a=2 \sqrt{\gamma / 3} \cos \theta / 3, \quad b=2 \sqrt{\gamma / 3} \cos (\theta+4 \pi) / 3, \quad c$ $=2 \sqrt{\gamma / 3} \cos (\theta+2 \pi) / 3$, and $k^{2}=(a-b) /(a-c)$ for $0<k$ $<1$. Here $\theta$ is given by $h=2(\gamma / 3)^{3 / 2} \cos \theta$ for $0<\theta<\pi$. This solution is periodic with period $L$ if

$$
4 K(k)=L \sqrt{2(a-c)} .
$$

When a stationary solution is required to be periodic, only one parameter ( $\gamma$ or $h$, we choose $\gamma$ ) is independent and the other parameter is determined by Eq. (21). A stationary periodic solution is shown in Fig. 2(a) for $\gamma=0.5$.

From the stationary periodic solutions, we can construct new periodic solutions by using the method described in Sec. II. The AKNS system (2) for the KdV equation is given by [3]

$$
\begin{gathered}
r=-1, \quad A=-4 \eta^{3}-2 \eta q-q_{x}, \\
B=-4 \eta^{2} q-2 \eta q_{x}-q_{x x}-2 q^{2}, \quad C=4 \eta^{2}+2 q .
\end{gathered}
$$

From Eq. (4), we have 


$$
\sigma(x)=\frac{\sqrt{2(a-c)}}{\left(4 \eta^{2}+2 a\right)(a-c)} \Pi(\phi, m, k),
$$

where $m=2(a-b) /\left(4 \eta^{2}+2 a\right)$, and

$$
\phi=\operatorname{am}\left(\frac{1}{2} \sqrt{2(a-c)} x, k\right)
$$

We also have, from Eq. (6),

$$
\alpha(\eta)=16 \eta^{6}-4 \eta^{2} \gamma+2 h .
$$

We further have, from Eq. (7),

$$
\Gamma=-\frac{1}{4 \eta^{2}+2 q(x)}\left[f(\xi)+4 \eta^{3}+2 \eta q(x)+q^{\prime}(x)\right],
$$

where $f(\xi)$ is given in Eq. (8).

The Bäcklund transformation for the $\mathrm{KdV}$ equation is [3]

$$
\tilde{q}(x, t)=q(x)-2 \Gamma_{x} .
$$

Therefore, the new periodic solutions can be written as

$$
\begin{aligned}
\tilde{q}(x, t)= & q(x)-\frac{q^{\prime}(x)}{\left[2 \eta^{2}+q(x)\right]^{2}}\left[f(\xi)+4 \eta^{3}+2 \eta q(x)\right. \\
& \left.+q^{\prime}(x)\right]+\frac{1}{2\left[2 \eta^{2}+q(x)\right]^{2}}\left\{-f^{\prime}(\xi)\right. \\
& \left.+2\left[2 \eta^{2}+q(x)\right]\left[2 \eta q^{\prime}(x)+q^{\prime \prime}(x)\right]\right\},
\end{aligned}
$$

where $f(\xi)$ [and its derivative $f^{\prime}(\xi)$ ] is given in Eq. (8), and $\eta$ satisfies either condition (9) or (11).

The asymptotic behavior for $t \rightarrow \pm \infty$ can be given analytically for the cases of $\alpha(\eta)>0$ and $\alpha(\eta)=0$. When $\alpha(\eta)$ $>0$,

$$
\begin{aligned}
\lim _{t \rightarrow \pm \infty} \tilde{q}(x, t)= & \frac{-2 \eta^{2} q^{2}(x)+\left(4 \eta^{4}-\gamma\right) q(x)+2 \eta^{2} \gamma-2 h}{\left[2 \eta^{2}+q(x)\right]^{2}} \\
& -\frac{\omega q^{\prime}(x)}{\left[2 \eta^{2}+q(x)\right]^{2}} \\
& \times \frac{\sinh \left[\omega \sigma(x)+c_{0}\right] \mp \cosh \left[\omega \sigma(x)+c_{0}\right]}{\cosh \left[\omega \sigma(x)+c_{0}\right] \pm \sinh \left[\omega \sigma(x)+c_{0}\right]} \\
& \text { for } \alpha(\eta)>0 .
\end{aligned}
$$

So, the asymptotic behavior when $t \rightarrow \infty$ is different from that when $t \rightarrow-\infty$, and the new solution is a kink solution. On the other hand, when $\alpha(\eta)=0$, asymptotic behaviors when $t \rightarrow \infty$ and when $t \rightarrow-\infty$ are both dominated by the same term $1 /\left[\sigma(x)-t+c_{0}\right]^{2}$, and therefore are the same, i.e.,

$$
\begin{aligned}
& \lim _{t \rightarrow \pm \infty} \tilde{q}(x, t) \\
&=\frac{-2 \eta^{2} q^{2}(x)+\left(4 \eta^{4}-\gamma\right) q(x)+2 \eta^{2} \gamma-2 h}{\left[2 \eta^{2}+q(x)\right]^{2}} \\
& \text { for } \alpha(\eta)=0 .
\end{aligned}
$$

So, the new solution is not a kink solution when $\alpha=0$. When $\alpha(\eta)<0$, the solution is a tangent function of time and may produce a profound effect on complicated dynamical behavior when the system is perturbed.

Figures 2(b) and 2(c) show solutions given by Eq. (27) for $\eta$ satisfying $\beta(\eta)=0$. The values of $\alpha$, calculated from Eq. (24), are real and greater than zero for both figures. [In our numerical study, we had not found a value of $\eta$ satisfying $\beta(\eta)=0$ that makes $\alpha(\eta) \leqslant 0$. If there exists such a negative $\alpha(\eta)$, then the solution contains tangent functions which may lead to a singular solution.] As expected, asymptotic behavior of the solutions when $t \rightarrow \infty$ and when $t \rightarrow-\infty$ are different from each other. Figure 2(b) is obtained when $\gamma$ $=0.5$. Two stationary solitons (or, call them "cavitons") coexist in time. The $x$ dependence of the solution changes gradually near $t=0$. Figure 2(c) is for $\gamma=10$. Only one stationary soliton exists at a time. The (spatial) location of the soliton changes abruptly at $t=0$. The solutions shown in Figs. 2(b) and 2(c) are new solutions. Their asymptotic behaviors when $t \rightarrow+\infty$ are different from those when $t \rightarrow$ $-\infty$. The new solutions correspond to new types of homoclinic orbits.

\section{THE MKdV EQUATION}

Now we consider the MKdV equation

$$
q_{t}+6 q^{2} q_{x}+q_{x x x}=0, \quad 0 \leqslant x \leqslant L, \quad-\infty<t<\infty
$$

where $q(x, t)$ is a real function. As shown in Appendix $\mathrm{C}$, the equation has the following stationary solutions characterized by two parameters, $\gamma$ and $h>-(3 / 2)(\gamma / 2)^{4 / 3}$ :

$$
q(x)=\frac{\left(q_{2} U-q_{1} V\right) \operatorname{cn}(\sqrt{U V} x, k)+q_{1} V+q_{2} U}{(U-V) \operatorname{cn}(\sqrt{U V} x, k)+U+V},
$$

where $\quad q_{1}=\frac{1}{2}\left(-b+\sqrt{-b^{2}-4 \gamma / b}\right), \quad q_{2}=\frac{1}{2}(-b$ $\left.-\sqrt{-b^{2}-4 \gamma / b}\right), \quad U=\left(b^{2}-b \sqrt{-b^{2}-4 \gamma / b}-2 \gamma / b\right)^{1 / 2}, \quad V$ $=\left(b^{2}+b \sqrt{-b^{2}-4 \gamma / b}-2 \gamma / b\right)^{1 / 2}, \quad$ and $\quad k=\left[\frac{1}{2}\right.$ $\left.-3 b^{2} /(4 U V)\right]^{1 / 2}$ for $0<k<\frac{1}{2}$. Here $b=-\left\{\left[2 \gamma^{2}\right.\right.$ $\left.\left.+\sqrt{4 \gamma^{4}+(4 h / 3)^{3}}\right]^{1 / 3}+\left[2 \gamma^{2}-\sqrt{4 \gamma^{4}+(4 h / 3)^{3}}\right]^{1 / 3}\right\}^{1 / 2}$. This solution is periodic with period $L$ if

$$
4 K(k)=L \sqrt{U V} .
$$

Figure 3(a) shows the stationary periodic solution for $\gamma=-0.05$. 
(a)

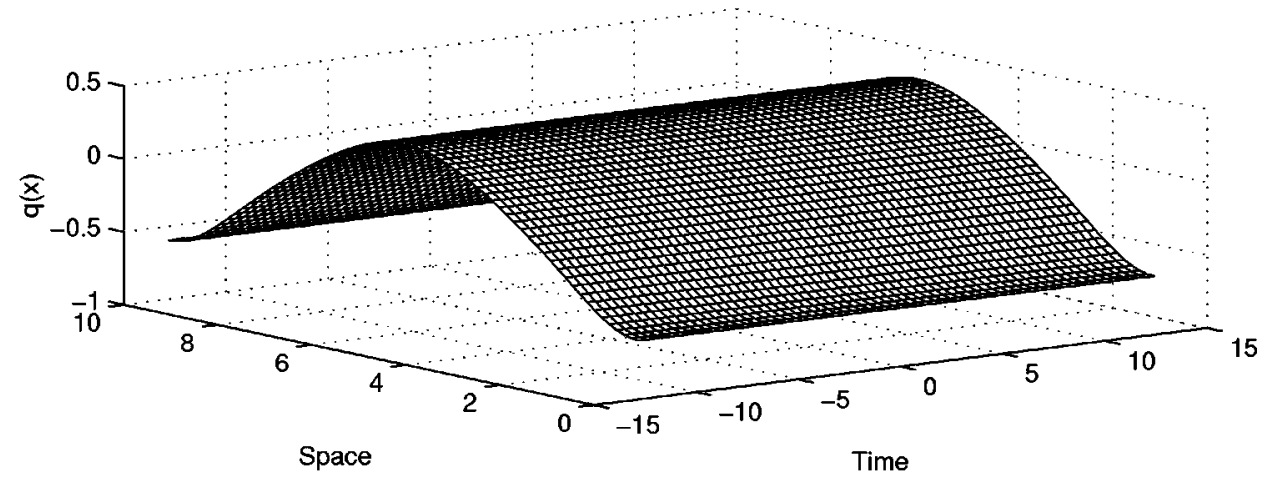

(b)

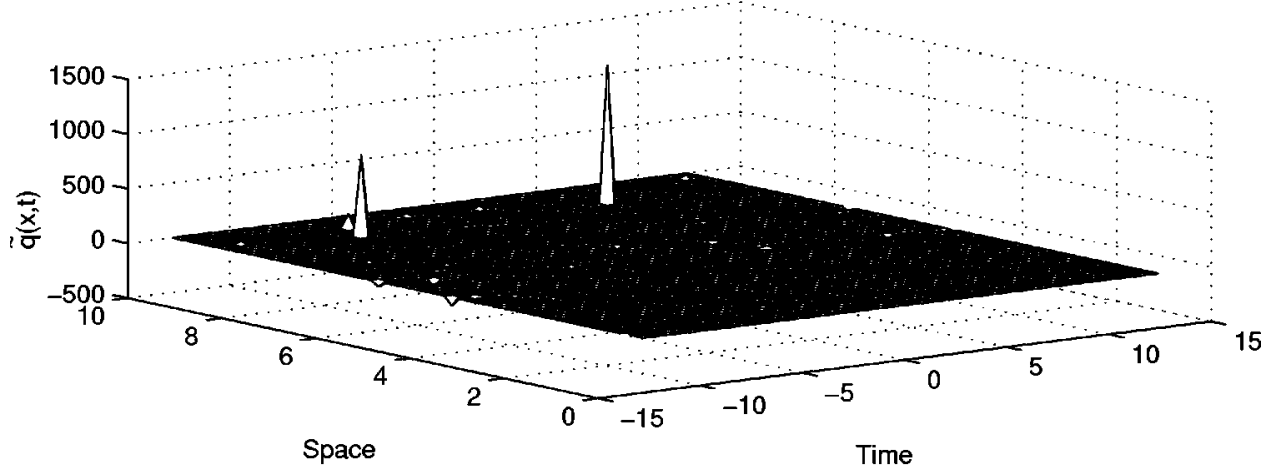

(c)

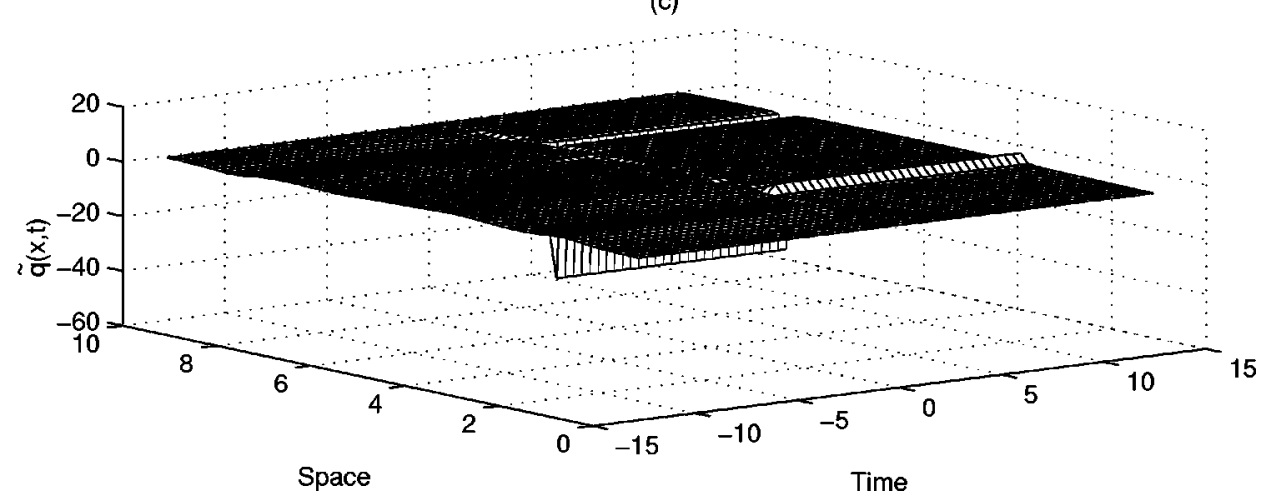

FIG. 3. The periodic solutions of the $\mathrm{MKdV}$ equation for the constant of integration $\gamma=-0.05$. (a) The stationary periodic solution. (b) The periodic solution constructed from the stationary periodic solution shown in (a) when $\eta= \pm 0.2074 . \alpha$ is real and $\alpha<0$. (c) Same as (b) but $\eta$ $= \pm 1.4539$ and $\alpha>0$.
The functions in the AKNS system for the MKdV equation are given by [3]

$$
r=-q, \quad A=-4 \eta^{3}-2 \eta q^{2}
$$

$$
\begin{gathered}
B=-q_{x x}-2 \eta q_{x}-4 \eta q^{2}-2 q^{3}, \\
C=q_{x x}-2 \eta q_{x}+4 \eta^{2} q+2 q^{3} .
\end{gathered}
$$

Thus,

$$
\sigma(x)=\int \frac{A_{1} \operatorname{cn}^{2}(\sqrt{U V} x, k)+A_{2} \operatorname{cn}(\sqrt{U V} x, k)+A_{3}}{A_{4} \operatorname{cn}^{2}(\sqrt{U V} x, k)+A_{5} \operatorname{cn}(\sqrt{U V} x, k)+A_{6} \operatorname{sn}(\sqrt{U V} x, k) \operatorname{dn}(\sqrt{U V} x, k)+A_{7}} d x,
$$

where $A_{1}=\left(q_{2} U-q_{1} V\right)(U-V), \quad A_{2}=2\left(q_{2} U-q_{1} V\right)(U$ $+V)+2\left(q_{1}-q_{2}\right) U V(U-V), \quad A_{3}=(U+V)^{2}+2\left(q_{1}\right.$ $\left.-q_{2}\right) U V(U+V), \quad A_{4}=\left[\gamma+4 \eta^{2}\left(q_{2} U-q_{1} V\right) /(U-V)\right](U$ $-V)^{2}, \quad A_{5}=2\left[\gamma+4 \eta^{2}\left(q_{2} U-q_{1} V\right) /(U-V)\right]\left(U^{2}-V^{2}\right)$ $+8 \eta^{2}\left(q_{1}-q_{2}\right) U V(U-V), \quad A_{6}=-2 \eta(U-V) \sqrt{U V}$, and $A_{7}=\left[\gamma+4 \eta^{2}\left(q_{2} U-q_{1} V\right) /(U-V)\right](U+V)^{2}+8 \eta^{2}\left(q_{1}\right.$ $\left.-q_{2}\right) U V(U+V)$. Further, from Eq. (6),

$$
\alpha(\eta)=16 \eta^{6}-\gamma^{2}-4 \eta^{2} h
$$

where $h$ depends on $\gamma$ due to Eq. (32).

The Bäcklund transformation for the MKdV equation is [3] 


$$
\tilde{q}(x, t)=q(x)-\frac{\Gamma_{x}}{\left(1+\Gamma^{2}\right)} .
$$

From Eq. (7), we have

$$
\Gamma=-\left[\gamma-2 \eta q^{\prime}(x)+4 \eta^{2} q(x)\right]\left[f(\xi)+4 \eta^{3}+2 \eta q^{2}(x)\right],
$$

and therefore

$$
\begin{aligned}
\Gamma_{x}= & {\left[2 \eta q^{\prime \prime}(x)-4 \eta^{2} q^{\prime}(x)\right]\left[f(\xi)+4 \eta^{3}+2 \eta q^{2}(x)\right] } \\
& -\left[\gamma-2 \eta q^{\prime}(x)+4 \eta^{2} q^{\prime \prime}(x)\right] \\
& \times\left[\frac{q(x)}{\gamma-2 \eta q^{\prime}(x)+4 \eta^{2} q(x)} f^{\prime}(\xi)+4 \eta q(x) q^{\prime}(x)\right],
\end{aligned}
$$

where $f(\xi)$ is given in Eq. (8). If one can find a value of $\eta$ satisfying either Eq. (9) or Eq. (11), then a new solution is obtained by substituting the expressions for $\Gamma$ and $\Gamma_{x}$ into Eq. (36).

Figures 3(b) and 3(c) show the new solutions when $\eta$ is required to satisfy $\beta(\eta)=0$. Figure $3(\mathrm{~b})$ is for $\alpha<0$. It is interesting to observe that rational solitons appear randomly in space and time. Figure 3(c) is for $\alpha>0$, where the solution is oscillatory when $t<0$ and is solitary when $t>0$. The pattern change occurs abruptly at $t=0$ but no rational soliton appears. The new solutions shown in Figs. 3(b) and 3(c) have the feature that their asymptotic behaviors when $t \rightarrow$ $+\infty$ are different from those when $t \rightarrow-\infty$. They correspond to new types of homoclinic orbits.

\section{CONCLUSIONS}

For a integrable nonlinear evolution equation with the periodic boundary condition, we use the Bäcklund transformation to construct a periodic solution from the stationary periodic solution. We give explicit conditions which should be satisfied by the constant $\eta$ needed in the transformation due to the required periodicity. The function $\Gamma$ needed in the transformation to construct a periodic solution is also given explicitly. Hence we are able to give the analytical expression of the newly constructed solution. The time dependence of the new solution can be the inverse, the tangent, the hyperbolic tangent, or a combination of exponentials.

We apply this method to the NLS, the $\mathrm{KdV}$, and the $\mathrm{MKdV}$ equation. The periodic solutions are given analytically. They are new because they were not explicitly written down before. Numerical visualizations of the new solutions are also given. All new solutions we obtained are kink solutions. Therefore a pattern change should occur in a time (near $t=0$ ). The change can be gradual or abrupt, and can be accompanied by the appearance of rational solitons distributed randomly in space and time. These new periodic solutions correspond to new types of homoclinic orbits due to different asymptotic behaviors in time.

\section{ACKNOWLEDGMENTS}

L.Z.R. and C.Y.L. are grateful to the Department of Mathematics of the Hong Kong University of Science and Technology for their hospitality. This work was partially supported by the Research Grant of Hong Kong under Grant No. HKUST606/95P, and by National Natural Science Foundation of China.

\section{APPENDIX A: THE STATIONARY PERIODIC SOLUTION OF THE NLS EQUATION}

A stationary solution of the NLS equation (12) can be written as $q(x)=\rho(x) e^{i \phi(x)}$, where the amplitude function $\rho(x)$ and the phase function $\phi(x)$ are real functions satisfying

$$
\begin{gathered}
\rho_{x x}-\rho\left(\phi_{x}\right)^{2}+2 \rho^{3}=0, \\
2 \rho_{x} \phi_{x}+\rho \phi_{x x}=0 .
\end{gathered}
$$

The second equation can be written as $\rho \sqrt{\phi_{x}}=\gamma$, where $\gamma$ is a constant of integration. Therefore the $\rho$ motion is given by $\rho_{x x}-\gamma^{4} / \rho^{3}+2 \rho^{3}=0$. Equivalently, the $\rho$ motion is governed by the Hamiltonian

$$
H\left(\rho, \rho_{x}\right)=\frac{1}{2} \rho_{x}^{2}+\frac{\gamma^{4}}{2 \rho^{2}}+\frac{1}{2} \rho^{4}=\frac{h}{2},
$$

where $h$ is a constant of motion. Hence, there are two centers, at $\left(\rho, \rho_{x}\right)=\left(\left(\gamma^{4} / 2\right)^{1 / 6}, 0\right)$ and $\left(-\left(\gamma^{4} / 2\right)^{1 / 6}, 0\right)$. When $h$ $>h_{0}=3\left(\gamma^{4} / 2\right)^{2 / 3}$, periodic orbits exist in the neighborhood of the centers. Let $u=\rho^{2} / \sqrt{2}$, then Eq. (A3) becomes

$$
\frac{1}{2} u_{x}^{2}+\gamma^{4}+u^{3}=h u
$$

We write this equation as

$$
u_{x}^{2}=-2(u-a)(u-b)(u-c),
$$

where $a, b$, and $c$ are roots of $u_{x}^{2}=-2\left(u^{3}+h u-\gamma^{4}\right)$. Explicitly, we have $a=2 \sqrt{h / 3} \cos (\theta / 3), b=2 \sqrt{h / 3} \cos [(4 \pi+\theta) / 3]$, $c=2 \sqrt{h / 3} \cos [(2 \pi+\theta) / 3]$, where $\theta$ is given by $-\gamma^{4}$ $=2(h / 3)^{3 / 2} \cos \theta$ for $\pi / 2<\theta<\pi$. Therefore, the amplitude function $\rho(x)$ is as given in Eq. (13) with modulus $k$ $=\sqrt{(a-b) /(a-c)}$ for $0<k<\frac{1}{2}$. The expression for the phase function $\phi(x)$, as given in Eq. (13), can be obtained from $\phi_{x}=\gamma^{2} / \rho^{2}$.

According to periodicity of the Jacobian elliptic functions, solution (13) is periodic if Eq. (14) is satisfied.

\section{APPENDIX B: THE STATIONARY PERIODIC SOLUTION OF THE KdV EQUATION}

A stationary solution of the $\mathrm{KdV}$ equation (19) satisfies

$$
3 q^{2}+q_{x x}=\gamma
$$

where $\gamma$ is a constant of integration. This equation is equivalent to Hamilton's equations of motion with the Hamiltonian 


$$
H=\frac{1}{2} p^{2}+q^{3}-\gamma q=h,
$$

where $p=q_{x}$ and $h$ is a constant. When $\gamma>0$ and $|h|<h_{0}$ $=2(\gamma / 3)^{3 / 2}$, this equation can be written as

$$
q_{x}^{2}=-2(q-a)(q-b)(q-c),
$$

where $a=2 \sqrt{\gamma / 3} \cos \theta / 3, \quad b=2 \sqrt{\gamma / 3} \cos (\theta+4 \pi) / 3$, and $c$ $=2 \sqrt{\gamma / 3} \cos (\theta+2 \pi) / 3$ are roots of $p^{2}=-2\left(q^{3}-\gamma q-h\right)$. Here $\theta$ is given by $h=2(\gamma / 3)^{3 / 2} \cos \theta$ for $0<\theta<\pi$. In the phase space, there is a center at $(q, p)=(\sqrt{\gamma / 3}, 0)$ and a saddle point at $(-\sqrt{\gamma / 3}, 0)$ for the Hamiltonian system. In the neighborhood of the center, Eq. (B3) has solution (20) with modulus $k=\sqrt{(a-b) /(a-c)}$ for $0<k<1$. The solution (20) takes $\gamma$ and $h$ as parameters.

According to periodicity of the Jacobian elliptic functions, solution (20) is periodic if Eq. (21) is satisfied.

\section{APPENDIX C: THE STATIONARY PERIODIC SOLUTION OF THE MKdV EQUATION} fies

The stationary solution of the MKdV equation (30) satis-

$$
q_{x x}+2 q^{3}=\gamma
$$

where $\gamma$ is a constant. This system is equivalent to the Hamiltonian system with the Hamiltonian

$$
H=\frac{1}{2} p^{2}+\frac{1}{2} q^{4}-\gamma q=\frac{h}{2}
$$

where $h$ is a constant. In the phase space, there exists only one center, at $(q, p)=\left((\gamma / 2)^{1 / 3}, 0\right)$. When $h>h_{0}=-(3 /$ $2)(\gamma / 2)^{4 / 3}$, there is a family of periodic orbits surrounding the center.

To find the solution of the Hamiltonian system, we write Eq. (C2) as

$$
q_{x}^{2}=\left(q-q_{1}\right)\left(q-q_{2}\right)\left(q-q_{3}\right)\left(q-q_{4}\right)
$$

where $\quad q_{1}=\frac{1}{2}\left(-b+\sqrt{b^{2}-4 c_{1}}\right), \quad q_{2}=\frac{1}{2}\left(-b-\sqrt{b^{2}-4 c_{1}}\right)$, $q_{3}=\frac{1}{2}\left(-b+i \sqrt{4 c_{2}-b^{2}}\right), \quad$ and $\quad q_{4}=\frac{1}{2}\left(-b-i \sqrt{4 c_{2}-b^{2}}\right)$ are roots of $p^{2}=-\frac{1}{2} q^{4}+\gamma q+h$. Here $b=\left\{\left[2 \gamma^{2}\right.\right.$ $\left.\left.+\sqrt{4 \gamma^{4}+(4 h / 3)^{3}}\right]^{1 / 3}+\left[2 \gamma^{2}-\sqrt{4 \gamma^{4}+(4 h / 3)^{3}}\right]^{1 / 3}\right\}^{1 / 2}, \quad c_{1}$ $=b^{2} / 2+\gamma / b$, and $c_{2}=b^{2} / 2-\gamma / b$. Equation (C3) has solution (31) with modulus $k=\left[\frac{1}{2}-3 b^{2} /\left(4 \sqrt{2 b^{4}+4 \gamma^{2} / b^{2}}\right)\right]^{1 / 2}$ for $0<k<\frac{1}{2}$.

According to periodicity of the Jacobian elliptic functions, solution (31) is periodic if Eq. (32) is satisfied.
[1] M.C. Cross and P.C. Hohenberg, Rev. Mod. Phys. 65, 851 (1993).

[2] M.J. Ablowitz and P.A. Clarkson, Solitons, Nonlinear Evolutions and Inverse Scattering (Cambridge University Press, Cambridge, England, 1991).

[3] K. Konno and M. Wodati, Prog. Theor. Phys. 53, 1652 (1975).

[4] C. Rogers and W.E. Shadwick, Bücklund Transformations and Their Application (Academic Press, New York, 1982).

[5] A.H. Khater, O.H. El-Kalaawy, and M.A. Helal, Chaos Solitons Fractals 8, 1901 (1997).

[6] J. Weiss, M. Tabor, and G. Garnevale, J. Math. Phys. 24, 522 (1983).

[7] A.H. Khater, Astrophys. Space Sci. 162, 151 (1989); A.H. Khater, M.F. El-Sabbath, and D.K. Callebaut, Comput. Math. Appl. 17, 1379 (1988).

[8] P.J. Ovlver, Application of Lie Groups to Differential Equations (Springer-Verlag, Berlin, 1986).

[9] W. Herman, A. Korpel, and P.P. Banerjee, Wave Motion 7,
283 (1985); W. Herman, P.P. Banerjee, and M.R. Chatterjee, J. Phys. A 22, 241 (1989); W. Herman and M.J. Takaoka, ibid. 23, 4805 (1990).

[10] W. Malfliet, J. Phys. A 24, 5499 (1991); Am. J. Phys. 60, 650 (1992); J. Phys. A 26, L723 (1993).

[11] C. Li and S. Wiggins, Invariant Manifolds and Vibrations for Perturbed Nonlinear Schrödinger Equations (Springer-Verlag, New York, 1997); Y. Li, D.W. McLaughlin, J. Shatah, and S. Wiggins, Commun. Pure Appl. Math. 49.11, 1175 (1996); Y. Li and D.W. McLaughlin, J. Nonlinear Sci. 7.3, 211 (1997).

[12] D.W. McLaughlin, E.A. Overman II, S. Wiggins, and C. Xiong, in Dynamics Reported -Expositions in Dynamical Systems, New Series Vol. 5, edited by C. K. R. T. Jones, U. Kirchgarder, and H. O. Wolther (Springer-Verlag, New York, 1996), pp. 190-237.

[13] N.N. Akhmediev and A. Ankiewicz, Solitons, Nonlinear Pulses and Beams (Chapman \& Hall, London, 1997). 